\section{Food Allergy in Adults: An Over- or Underrated Problem?}

by Dr. med. Cornelia S. Seitz, Petra Pfeuffer, Petra Raith, Prof. Dr. med. Eva B. Bröcker, PD Dr. med. Axel Trautmann in volume 42/2008

\section{Stringent Elimination Diet}

Seitz et al conducted a retrospective analysis of more than 400 patients with suspected food allergies and rightly emphasize that "only a comprehensive allergological evaluation" can protect patients from the negative consequences of over- or underestimating the food allergy (1). In 66 cases they describe oral provocation tests-following a medical history and standardized skin tests-whose results were negative. The authors diagnose a food allergy in more than $50 \%$ of their patients - but we don't know whether in case the situation was not obvious, oral provocation tests were conducted in these patients too.

In everyday practice, the proportion of patients with unclear constellations after a medical history has been taken, skin tests have been conducted, or IgE concentrations were measured is high (for example, existing differential diagnoses to food allergies and sensitization to several foodstuffs in unequivocal symptoms after a composite meal). The food allergy working group of the three German allergy societies (the German Society for Allergology and Clinical Immunology [DGAKI], the Medical Association of German Allergologists [ÄDA,], and the Society of Pediatric Allergology [GPA]) have compiled position papers and guidelines relevant to clinical practice that can be found at http://www.leitlinien.net by searching for "food allergy". The specialist societies are particularly concerned that institutions with allergological experience should extensively offer the option of oral provocation tests for patients whose constellation is unclear. Targeted oral provocation tests can be sufficiently reproduced in hospitals under DRG conditions and are often the only option to confirm a suspected diagnosis of food allergy (2, 3).

Food allergies can have harmless outcomes but they can also be fatal, albeit rarely. Neither the degree of sensitization nor the extent of a previous systemic reaction are predictive of the outcome of life threatening reactions to foodstuffs. Hence even patients with "mild" confirmed systemic reactions to foodstuffs should stringently adhere to a strict elimination diet after dietary advice and should carry an emergency kit (adrenaline auto injector, antihistamine, steroid) on their person.

\section{REFERENCES}

1. Seitz CS, Pfeuffer P, Raith P, Bröcker E-B, Trautmann A:Food Allergy in Adults: An Over- or Underrated Problem?.Dtsch Arztebl Int 2008; 105(42): 715-23.
2. Niggemann B, Erdmann S, Jäger L et al.: Standardisierung von oralen Provokationstests bei IgE-vermittelten Nahrungsmittelallergien. Aktualisierte Leitlinie von DGAKI, GPA und ÄDA. Allergo J 2006; 14: 262-70.

3. Henzgen M, Vieths S, Reese I et al.: Nahrungsmittelallergien durch immunologische Kreuzreaktionen. Leitlinie der DGAKI und des ÄDA. Allergo J 2005; 14: 48-59.

Prof. Dr. med. Thomas Werfel

Klinik für Dermatologie, Allergologie und Venerologie

Medizinische Hochschule Hannover

Ricklinger Str. 5

30449 Hannover, Germany

Werfel.Thomas@mh-hannover.de

\section{Dr. med. Ute Lepp}

Herz-Lungenpraxis Stade

Harsefelder Str. 6

21680 Stade, Germany

info@herzlunge.de

Prof. Dr. med. Margitta Worm

Allergie-Centrum-Charité

Klinik für Dermatologie, Venerologie und Allergologie

Charité Campus Mitte

Universitätsmedizin Berlin

Charitéplatz 1

10117 Berlin, Germany

margitta.worm@charite.de

\section{In Reply:}

In principle we have nothing to add to the correspondence, which primarily refers to the guidelines for the diagnosis of food allergies. However, in this context the advantages/problems of guidelines - which we actually cited (1)—should not remain unmentioned. On the one hand these are often published in journals to which many potentially interested parties do not have access. On the other hand, if one searches for answers to concrete questions-often those of particular importance-one often finds only a discussion of different approachesunequivocal and practical recommendations for action are unfortunately rarely provided.

With regard to the provocation tests, we wish to point out that our study was confined to the diagnosis of IgE mediated (type I) food allergy with typical immediate symptoms (oral allergy syndrome, urticaria/angioedema, anaphylaxis). Simple methods were the main focus, including history taking and skin prick tests. Additional laboratory tests (serum IgE, tryptase) and/or provocation tests take time; and, especially in IgE mediated allergies, provocation tests entail substantial risks. Rational selection and interpretation of diagnostic methods in individual patients depend on symptoms (anaphylaxis or oral allergy syndrome), the suspected foodstuff (common or rare allergen), and should take into considerations sensitivity and specificity of the test method. In obvious symptoms or oral allergy syndrome or anaphylaxis in connection with ingestion of a known food allergen for which IgE sensitization was confirmed by skin test, no provocation test is necessary $(2,3)$. This is in complete contrast to suspected deterioration of atopic eczema after ingestion 
of certain foodstuffs, a scenario in which patients should undergo a provocation test with a positive result before starting a diet-but our study did not include any such patients.

DOl: 10.3238/arztebl.2009.0116

\section{REFERENCES}

1. Seitz CS, Pfeuffer P, Raith P, Bröcker E-B, Trautmann A:Food Allergy in Adults: An Over- or Underrated Problem?.Dtsch Arztebl Int 2008; 105(42): 715-23.

2. Trautmann A: Nahrungsallergie. In: Trautmann A: Allergiediagnose, Allergietherapie. Stuttgart, New York: Thieme 2006; 181-201.
3. Bernstein IL et al.: Food allergy: a practice parameter. Ann Allergy Asthma Immunol 2006; 96: 1-68.

\section{Priv.-Doz. Dr. med. Axel Trautmann}

Klinik und Poliklinik für Dermatologie, Venerologie und Allergologie der Universität Würzburg

Josef-Schneider-Str. 2, 97080 Würzburg, Germany

trautmann_a@klinik.uni-wuerzburg.de

Conflict of interest statement

The authors of the letter and of the reply declare that no conflict of interest exists according to the guidelines of the International Committee of Medical Journal Editors. 